PROCEEDINGS OF THE

AMERICAN MATHEMATICAL SOCIETY

Volume 139, Number 10, October 2011, Pages 3623-3632

S 0002-9939(2011)10891-8

Article electronically published on February 28, 2011

\title{
ON DISPLACEMENT INTERPOLATION OF MEASURES INVOLVED IN BRENIER'S THEOREM
}

\author{
NICOLAS JUILLET
}

(Communicated by Mario Bonk)

\begin{abstract}
We prove that in the Wasserstein space built over $\mathbb{R}^{d}$ the subset of measures that does not charge the non-differentiability set of convex functions is not displacement convex. This completes the study of Gigli on the geometric structure of measures meeting the sharp hypothesis of the refined version of Brenier's Theorem.
\end{abstract}

Optimal transport is nowadays a central tool in many fields of analysis, differential geometry and probability theory (see e.g. the books by Villani [11, 12]). It takes its origin in the problem of Monge, asking for the shortest way to displace an amount of soil from one place of the Euclidean space to a heap of soil at another place. This problem induces a very natural distance between probability measures - the Wasserstein distance - where the measures represent the heaps of soil. Wasserstein distance is somewhat complementary with the Lebesgue $L^{p}$ norms because it corresponds to a "horizontal" displacement: in the first approximation, for two localized probability measures on $\mathbb{R}^{d}$, the Wasserstein distance is the distance between their barycenters.

Brenier's Theorem [4] on monotone rearrangement of maps of $\mathbb{R}^{d}$ has become the very core of the theory of optimal transport. It gives a representation of the optimal transport map in terms of gradient of convex functions. A very enlightening heuristic on $\left(\mathcal{P}_{2}\left(\mathbb{R}^{d}\right), W_{2}\right)$ is proposed in 7 , where it appears with an infinite differential structure and the Wasserstein distance is seen as a Riemannian-like distance. This point of view has created many developments, among which is the gradient flow theory presented in [2]. In [6], Gigli explores the Riemannian-like structure and proposes to think of the measures meeting the hypothesis of a refined version of Brenier's Theorem as the regular points of the Wasserstein space. In this paper we show that the set of those transport-regular measures is not geodesically convex (Theorems 2.2 and 2.5) . It is quite surprising because it is well-known that the subset made of absolutely continuous measures (the most notorious transportregular measures) is geodesically convex. It answers a question suggested by Gigli [6. Remark 2.12].

The paper is organized as follows: we first recall some main results on the quadratic Monge(-Kantorovich) problem. The key idea of our result can be found in Lemma 1.6. while Proposition 1.8 provides a way, together with Proposition 1.7

Received by the editors July 23, 2010 and, in revised form, August 31, 2010.

2010 Mathematics Subject Classification. Primary 28A75.

Key words and phrases. Optimal transport, Wasserstein space, rectifiability.

(C)2011 American Mathematical Society 
to characterize transport-regular measures. In the second (and last) part, we prove the main theorem (Theorem 2.2) and its generalization (Theorem 2.5), thanks to explicit constructions.

Remark 0.1. After the paper was submitted, Shin-Ichi Ohta explained to the author that Theorem 2.2 together with [6, Corollary 6.6], shows that on $\left(\mathcal{P}_{2}\left(\mathbb{R}^{d}\right), W_{2}\right)$, which is a non-negatively curved space (see [12, Bibliographic notes of Chapter $26]$ ), there is a geodesic curve $\gamma$ such that $\gamma(0), \gamma(1)$ are regular and the mid-point $\gamma(1 / 2)$ is non-regular in the sense of Alexandrov spaces. This phenomenon can only occur in infinite dimensional spaces. Indeed it was proven in 10, Corollary of Theorem 1.2(A), p. 132] that in finite dimensional Alexandrov spaces with curvature bounded below the set of regular points (i.e., those with a Euclidean space as tangent cone) is geodesically convex.

\section{The Quadratic Monge-Kantorovich problem in $\mathbb{R}^{d}$}

1.1. The problem. We denote by $\mathcal{P}_{2}\left(\mathbb{R}^{d}\right)$ the Wasserstein space on $\mathbb{R}^{d}$, i.e., the space of Borel probability measures $\mu$ on $\mathbb{R}^{d}$ such that $\int|x|^{2} \mathrm{~d} \mu(x)<\infty$. Let $\mu_{0}, \mu_{1} \in \mathcal{P}_{2}\left(\mathbb{R}^{d}\right)$. We consider

$$
C(\pi)=\iint_{\mathbb{R}^{d} \times \mathbb{R}^{d}}|p-q|^{2} \mathrm{~d} \pi(p, q),
$$

where $\pi \in \mathcal{P}_{2}\left(\mathbb{R}^{d} \times \mathbb{R}^{d}\right)$ has first marginal $e_{0 \#} \pi=\mu_{0}$ and second marginal $e_{1 \#} \pi=$ $\mu_{1}$. Here $e_{0} \otimes e_{1}=\operatorname{Id}_{\mathbb{R}^{d} \times \mathbb{R}^{d}}$. We say that $\pi$ is a transport plan (or a coupling) between $\mu_{0}$ and $\mu_{1}$ if it satisfies the conditions on marginals. The Monge-Kantorovich problem that we consider here consists in minimizing $C(\pi)$ among all transport plans (see [12 or 2] for generalizations of this problem). The minimizers in (1.1) are called optimal transport plans. In this setting, it is well-known that there exists at least one optimal transport plan (see e.g. 11, Proposition 2.1]). The Wasserstein distance between $\mu_{0}$ and $\mu_{1}$ is then $W_{2}\left(\mu_{0}, \mu_{1}\right)=\sqrt{C(\pi)}$, where $\pi$ is a minimizer. Among the questions of interest are the uniqueness and the properties of the optimal transport plans. These questions are correlated. Actually, if we can prove some special property for minimizers of (1.1), we can restrict the set of candidates and may obtain uniqueness. A key feature of an optimal transport plan is the cyclical monotonicity of its support (which actually is a property that characterizes optimal plans). It will be described in subsection 1.2. Continuing the analysis of the support of optimal $\pi$ 's can lead to deterministic couplings, the so-called transport maps presented in subsection 1.3 .

1.2. Cyclical monotonicity. We first review some basic results. The problem (1.1) can be somewhat discretized by introducing the cyclically monotone sets.

Definition 1.1. A set $S \subset \mathbb{R}^{d} \times \mathbb{R}^{d}$ is said to be cyclically monotone if and only if for any $n \in \mathbb{N}^{*}$ and any permutation $\sigma \in \Sigma_{n}$,

$$
\sum_{i=1}^{n}\left|x_{k}-y_{k}\right|^{2} \leq \sum_{k=1}^{n}\left|x_{k}-y_{\sigma(k)}\right|^{2}
$$

where $\left(x_{k}, y_{k}\right) \in S$ for $k=1, \ldots, n$ is arbitrary.

The next result provides a geometric characterization of optimal transport plans. 
Proposition 1.2. Let $\pi \in \mathcal{P}_{2}\left(\mathbb{R}^{d} \times \mathbb{R}^{d}\right)$. Then $\pi$ is an optimal transport plan between $\mu_{0}=e_{0 \#} \pi$ and $\mu_{1}=e_{1 \#} \pi$ if and only if $\operatorname{Spt}(\pi)$ is cyclically monotone.

A proof can be founded in [2, Theorem 6.1.4]. See also the comments in Chapter 5 of [12] for different general assumptions.

Note that it is not easy to check whether a particular transport plan is cyclically monotone. One can sometimes identify that it is not, thanks to the next classical fact.

Corollary 1.3 (Cycles of length 2). Let $\pi \in \mathcal{P}_{2}\left(\mathbb{R}^{d} \times \mathbb{R}^{d}\right)$ be an optimal transport plan. Then for any $(x, y)$ and $\left(x^{\prime}, y^{\prime}\right)$ in $\operatorname{Spt}(\pi)$ we have

$$
\left\langle\overrightarrow{x x^{\prime}}, \overrightarrow{y y^{\prime}}\right\rangle \geq 0 .
$$

Proof. Let $(x, y)$ and $\left(x^{\prime}, y^{\prime}\right)$ be in $\operatorname{Spt}(\pi)$. Proposition 1.2 indicates that $|x-y|^{2}+$ $\left|x^{\prime}-y^{\prime}\right|^{2} \leq\left|x-y^{\prime}\right|^{2}+\left|x^{\prime}-y\right|^{2}$. Writing $y^{\prime}-x=\left(y^{\prime}-y\right)+(y-x)$ as well as $y^{\prime}-x^{\prime}=\left(y^{\prime}-y\right)+(y-x)$ we obtain

$$
\left\{\begin{array}{c}
2\left\langle y^{\prime}-y, y-x\right\rangle=\left|y^{\prime}-x\right|^{2}-\left|y^{\prime}-y\right|^{2}-|y-x|^{2} \\
2\left\langle y^{\prime}-y^{\prime}, y-x^{\prime}\right\rangle=\left|y^{\prime}-x^{\prime}\right|^{2}-\left|y^{\prime}-y\right|^{2}-\left|y-x^{\prime}\right|^{2} .
\end{array}\right.
$$

The result comes from the difference of these relations.

We will say that a measure $\pi \in \mathcal{P}\left(\mathbb{R}^{d} \times \mathbb{R}^{d}\right)$ is order-preserving if (1.3) is satisfied for any $(x, y)$ and $\left(x^{\prime}, y^{\prime}\right)$ in $\operatorname{Spt}(\pi)$.

1.2.1. Application of Corollary 1.3 to $d=1$. The next lemma is a variation of the well-known theory of optimal transport in dimension 1. It will be useful in the proof of Lemma 1.6, which is the main innovation of this paper. Note that a measure on $\mathbb{R} \times \mathbb{R}$ is order preserving if and only if $x<x^{\prime} \Rightarrow y \leq y^{\prime}$ for every $(x, y),\left(x^{\prime}, y^{\prime}\right) \in \operatorname{Spt}(\pi)$.

Lemma 1.4. For two measures $\mu_{0}$ and $\mu_{1}$ in $\mathcal{P}_{2}(\mathbb{R})$, there is a unique orderpreserving transport plan. It is uniquely determined by

$$
\pi(]-\infty, x] \times]-\infty, y])=\min \left(F_{0}(x), F_{1}(y)\right),
$$

where $\left.\left.F_{i}(t)=\mu_{i}(]-\infty, t\right]\right)$ is the cumulative distribution of $\mu_{i}$ for $i \in\{1,2\}$.

Remark 1.5. Thanks to Corollary 1.3. the optimal transport plans must be $\pi$.

Proof. It is an easy exercise to see that $\pi$ is the law of the random vector $(X, Y)$ with $X=F_{0}^{-1}(U), Y=F_{1}^{-1}(U)$, where $U$ is uniform on $[0,1]$ and $F_{i}^{-1}$ is the generalized inverse of $F_{i}$ for $i \in\{0,1\}$. Let us recall that

$$
F_{i}^{-1}(u)=\inf \{t \in \mathbb{R} \mid F(t) \geq u\} .
$$

Hence, as $F_{0}$ and $F_{1}$ are non-decreasing, $\pi$ is order-preserving.

Now let $\pi^{\prime}$ be an a priori different optimal transport plan for the same MongeKantorovich problem and let $\left.\left.\left.\left.H(s, t)=\pi^{\prime}(]-\infty, s\right] \times\right]-\infty, t\right]\right)$. We have to prove that $H(s, t)=\min \left(F_{0}(s), F_{1}(t)\right)$. The inequality $\leq$ is obvious because $F_{0}(s)=$ $\left.\left.\pi^{\prime}(]-\infty, s\right] \times \mathbb{R}\right)$ and $\left.\left.F_{1}(t)=\pi^{\prime}(\mathbb{R} \times]-\infty, t\right]\right)$. For the other estimation it is enough to prove $\left(F_{0}(s)>H(s, t)\right) \Rightarrow\left(F_{1}(t) \leq H(s, t)\right)$. Indeed one can treat $F_{1}(t)>H(s, t)$ in the same way. So we assume $F_{0}(s)>H(s, t)$, i.e., $\left.\left.\pi^{\prime}(]-\infty, s\right] \times\right] t,+\infty[)>0$. Let $(x, y) \in \operatorname{Spt}\left(\pi^{\prime}\right)$ so that $x \leq s$ and $y>t$. Then because of Corollary 1.3. $\operatorname{Spt}\left(\pi^{\prime}\right) \operatorname{does}$ not meet $] x,+\infty[\times]-\infty, y[\supset] s,+\infty[\times]-\infty, t]=: R$. Therefore $F_{1}(t)-H(s, t)=$ $\pi^{\prime}(R)=0$. 
1.2.2. Application of Corollary 1.3 to measures concentrated on curves. Let $\vec{u}$ be a unit vector of $\mathbb{R}^{d}$. We define a partial order on $\mathbb{R}^{d}$ by $p \preceq q$ if and only if

$$
\langle\vec{u}, \overrightarrow{p q}\rangle>|q-p| \cos \frac{\pi}{4} \quad \text { or } \quad p=q .
$$

Actually $\left\{q \in \mathbb{R}^{d} \mid p \preceq q\right\}$ is a half-cone directed by $\vec{u}$, with edge $p$ and opening angle $\frac{\pi}{4}$. It is easy to check that $\preceq$ is transitive and antisymmetric. We note that $p \prec q$ if $p \preceq q$ and $p \neq q$. As in subsection 1.2.1 we can reinterpret (1.3): let $\mu_{0}, \mu_{1}$ be two measures of $\mathbb{R}^{d}$ such that $\preceq$ is a total order on $\operatorname{Spt}\left(\mu_{i}\right)$ for $i=0,1$ and $(x, y),\left(x^{\prime}, y^{\prime}\right) \in \operatorname{Spt}\left(\mu_{0}\right) \times \operatorname{Spt}\left(\mu_{1}\right)$. If $x \prec x^{\prime}$ we have

$$
\overrightarrow{\left\langle x x^{\prime}, y y^{\prime}\right\rangle} \begin{cases}>0 & \text { if } y \prec y^{\prime}, \\ <0 & \text { if } y^{\prime} \prec y, \\ =0 & \text { if } y=y^{\prime} .\end{cases}
$$

Therefore, a transport plan $\pi$ between $\mu_{0}$ and $\mu_{1}$ is order-preserving if and only if $x \prec x^{\prime} \Rightarrow y \preceq y^{\prime}$ on $\operatorname{Spt}(\pi)$. Note that (1.5) is easy to check. For instance, in the case $y \prec y^{\prime}$ the angle between the vectors is smaller than $\frac{\pi}{2}=\frac{\pi}{4}+\frac{\pi}{4}$ (see Figure 1).

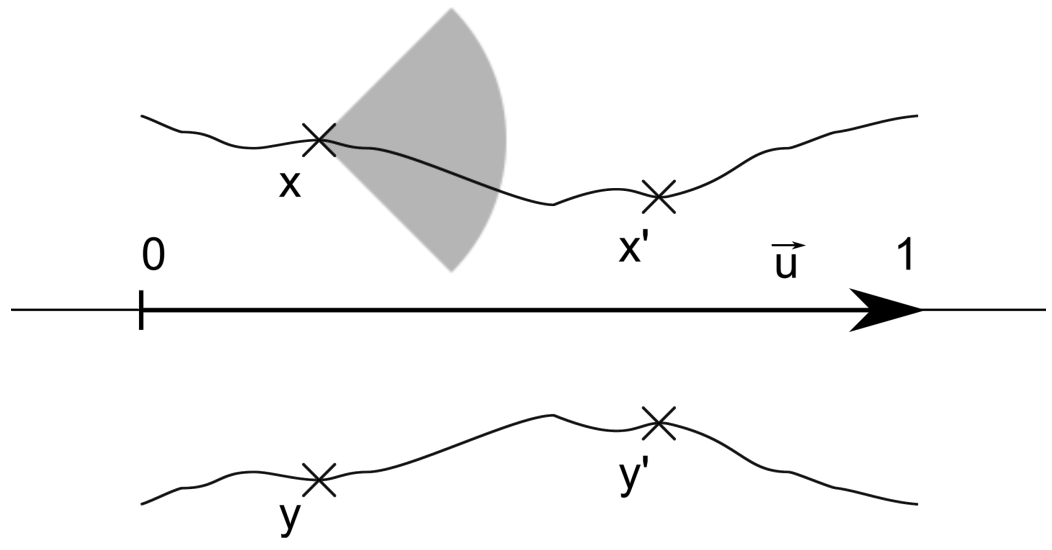

Figure 1. Totally ordered sets.

Lemma 1.6. Let $\vec{u}$ be a unit vector of $\mathbb{R}^{d}$. Assume that $\operatorname{Spt}\left(\mu_{0}\right)$ and $\operatorname{Spt}\left(\mu_{1}\right)$ are totally ordered with respect to $\vec{u}$. There is a unique order-preserving transport plan $\pi$ between $\mu_{0}$ and $\mu_{1}$. Therefore, thanks to Corollary 1.3, any optimal transport plan between $\mu_{0}$ and $\mu_{1}$ is $\pi$.

Proof. Let $\pi$ be an order-preserving transport plan between the $\mu_{i}$ 's. Let $p$ be the orthogonal projection on $L_{\vec{u}}$, the vectorial line directed by $\vec{u}$. The projection $p$ from $\left(\mathbb{R}^{d}, \preceq\right)$ to $\left(L_{\vec{u}}, \preceq\right)$ is strictly increasing so that because of Lemma 1.4. $\omega=(p \otimes p)_{\#} \pi$ is an order-preserving transport plan between $p_{\#} \mu_{0}$ and $p_{\#} \mu_{1}$ and it is the unique one. In fact, $\left(L_{\vec{u}}, \preceq, d_{\mathbb{R}^{d}}\right)$ and $\left(\mathbb{R}, \leq, d_{\mathbb{R}}\right)$ are isomorphic. But for $i \in\{0,1\}$ the projection $p$ is one-to-one from $\operatorname{Spt}\left(\mu_{i}\right)$ to its image so that we can deduce $\pi$ from $\omega$. Explicitly $\pi=\left(q_{0} \otimes q_{1}\right)_{\#} \omega$, where $q_{i}$ is the inverse map of $p: \operatorname{Spt}\left(\mu_{i}\right) \rightarrow L_{\vec{u}}$. 
Under the hypothesis of Lemma 1.6, with the notation above we have

$$
\pi\{(x, y) \mid\langle\vec{u}, \vec{x}\rangle \leq s ;\langle\vec{u}, \vec{y}\rangle \leq t\}=\min \left(\mu_{0}\{\langle\vec{u}, \vec{x}\rangle \leq s\}, \mu_{1}\{\langle\vec{u}, \vec{y}\rangle \leq t\}\right),
$$

and this expression determines uniquely $\pi$. This can be seen easily from Lemmas 1.4 and 1.6 and their proofs.

1.3. Necessary and sufficient condition for a Brenier map. The transport maps from $\mu_{0}$ to $\mu_{1}$ are maps $T: \mathbb{R}^{d} \rightarrow \mathbb{R}^{d}$ such that $T_{\#} \mu_{0}=\mu_{1}$. They are associated to a transport plan by $\pi_{T}=(\operatorname{Id} \otimes T)_{\#} \mu_{0}$. If $\pi_{T}$ is a minimizer of (1.1), $T$ is called an optimal transport map or Brenier map. The well-known theorem of Brenier ensures the existence of optimal maps under the assumption that $\mu_{0}$ is absolutely continuous w.r.t. the Lebesgue measure. In [5] this assumption has been weakened to $\mu_{0}$ gives 0 mass to Lipschitz hypersurfaces (also see the discussion below). The recent work by Gigli explores the sharpness of the hypothesis on $\mu_{0}$ permitting an optimal transport map for any $\mu_{1}$. Analytic and geometric characterizations for these measures are given in [6]. We will see in Theorems 2.2 and 2.5 that contrary to absolutely continuous measures, this set of measures is not stable under displacement interpolation.

The next result, especially implication $(2) \Rightarrow(1)$, is part of [6]. In what follows, all convex functions are real-valued.

Proposition 1.7 (Refined version of Brenier's Theorem). Let $\mu_{0} \in \mathcal{P}_{2}\left(\mathbb{R}^{d}\right)$. Then the following statements on $\mu_{0}$ are equivalent:

(1) Any convex function $\phi$ defined on a convex open $U \subset \mathbb{R}^{d}$ is $\mu_{0}$-a.e. differentiable.

(2) For any measure $\mu_{1} \in \mathcal{P}_{2}\left(\mathbb{R}^{d}\right)$, there exists a unique solution $\pi$ to the Monge-Kantorovich problem, and it is induced by a transport map $T$.

We'll call $\mu_{0}$ satisfying one of these statements a transport-regular measure (or simply a regular measure as in [6]). The map $T$ can be written as $\nabla \phi$ for some convex function $\phi$.

Statement (1) can be replaced by $\left(1^{\prime}\right)$ : Any convex function $\phi$ defined on $\mathbb{R}^{d}$ is $\mu_{0}$-a.e. differentiable. Assuming $\left(1^{\prime}\right)$, let $\phi$ be a convex function defined on $U$. We can cover $U$ by countably many open balls $B_{k}$ where the slope of $\phi$ is bounded. Then

$$
\overline{\phi_{k}}=\sup \left\{l(x) \mid l \text { is linear and } l \leq \phi \text { on } B_{k}\right\}
$$

is a convex function of $\mathbb{R}^{d}$ that coincides with $\phi$ on $B_{k}$. Each point of nondifferentiability of $\phi$ is a point of non-differentiability for some $\overline{\phi_{k}}$. It follows that $\phi$ is $\mu_{0}$-a.e. differentiable. Note that in (1), one can also add the points of $\partial U$ as points of non-differentiability. For that, consider $\partial U$ as the set of non-differentiability of the convex function $x \rightarrow d(x, U)$.

Gigli states (1) in a different way in terms of $\mathrm{c}-\mathrm{c}$ hypersurfaces. These objects appear in the work by Zajíček 13] in relation to the non-differentiability set of a convex function and for the first time in relation to optimal transport in a footnote of [5]. Actually Zajíček proved in [13] that the set of non-differentiability of a convex function is included in countably many $\mathrm{c}-\mathrm{c}$ hypersurfaces and that, conversely, such a union is exactly the set of non-differentiability of some function. We will not define $\mathrm{c}-\mathrm{c}$ hypersurfaces because we use a different result on the set of nondifferentiability points. Here is a weak formulation of a result due to Alberti [1]. 
Proposition 1.8 (Alberti). Let $\phi$ be a convex function of $\mathbb{R}^{d}$. There are $(d-1)$ dimensional submanifolds $M_{k} \subset \mathbb{R}^{d}$ of class $\mathcal{C}^{2}$, and a set $N$ with $\mathcal{H}^{d-1}(N)=0$ such that $\phi$ is differentiable outside $\bigcup_{k=1}^{+\infty} M_{k} \cup N$.

Example 1.9. We give some examples of transport-regular measures. They share the property to be defined by duality as the measures vanishing on special classes of sets $F$.

- If $\mu_{0}$ is absolutely continuous (i.e., $\mathcal{H}^{d}(F)=0 \Rightarrow \mu_{0}(F)=0$ ), then it is transport-regular. This assumption is the classical assumption of Brenier's Theorem on existence and uniqueness of minimizers in (1.1).

- If $\left(\operatorname{dim} \mathcal{H}(F) \leq d-1 \Rightarrow \mu_{0}(F)=0\right)$, then $\mu_{0}$ is transport-regular. This is the setting of the more usual refined Brenier Theorem, presented in [11, Theorem 2.32].

- A Lipschitz piece is any set $f(E)$ for some $E \subset \mathbb{R}^{d-1}$ and $f$ a Lipschitz continuous map. If

$-\mu_{0}$ is absolutely continuous with respect to $\mathcal{H}^{d-1}$ and

- $\mu_{0}(F)=0$ for every Lipschitz piece $F$ (i.e., $\mu_{0}$ is purely non-rectifiable), then $\mu_{0}$ is transport-regular. Any $\mathcal{C}^{1}$-hypersurface of $\mathbb{R}^{d}$ is contained in a countable union of Lipschitz pieces.

The measures in the proof of the main theorem are of the type described in the example below.

Example 1.10. If (i) $\mu_{0}$ is absolutely continuous with respect to $\mathcal{H}^{d-1}$ and (ii) it does not charge hypersurfaces of class $\mathcal{C}^{2}$ (i.e., $\mu_{0}$ is $\mathcal{C}^{2}$-purely non-rectifiable), then $\mu_{0}$ is transport-regular.

\section{Displacement interpolation of transport-REgular measures}

It is part of the theory to consider the displacement interpolation introduced by McCann in [9. Let $\mu_{0}, \mu_{1}$ be such that there exists a unique optimal plan $\pi$ between them. For any $t \in[0,1]$ we consider $\mu_{t}=e_{t \#} \pi$, where $e_{t}(x, y)=t x+(1-t) y$. For the unicity in the definition of $\mu_{t}$, we assume that the optimal transport plan $\pi$ is unique. If $\pi=(\operatorname{Id} \otimes T)_{\#} \mu_{0}$, we have $\mu_{t}=T_{t \#} \mu_{0}$, where $T_{t}=t T+(1-t)$ Id. The curve $\left(\mu_{t}\right)_{t \in[0,1]}$ defines the unique geodesic curve between $\mu_{0}$ and $\mu_{1}$ in $\left(\mathcal{P}_{2}\left(\mathbb{R}^{d}\right), W_{2}\right)$. We are interested in the question of whether $\mu_{t}$ is transport-regular for $t<1$. Because of Corollary 1.3, for $\mu_{0} \otimes \mu_{0}$-almost every $x, x^{\prime}$ we have

$$
\left\langle\overrightarrow{x x^{\prime}}, \overrightarrow{T_{t} x T_{t} x^{\prime}}\right\rangle \geq(1-t)\left|x^{\prime}-x\right|^{2},
$$

so that

$$
\left|T_{t} x^{\prime}-T_{t} x\right| \geq(1-t)\left|x^{\prime}-x\right| .
$$

Hence there exists a $\mu_{t}$-measurable map $S_{t}$ such that $\mu_{0}$-almost surely $S_{t} \circ T_{t}=\mathrm{Id}$. Therefore $S_{t \#} \mu_{t}=\mu_{0}$ and

$$
\left|y^{\prime} y\right| \geq \frac{1}{(1-t)}\left|\overrightarrow{S_{t} y^{\prime} S_{t} y}\right|
$$

for $\mu_{t} \otimes \mu_{t}$ almost every $\left(y, y^{\prime}\right)$. Then up to a modification of $\mu_{t}$-measure $0, S_{t}$ is Lipschitz continuous on $\operatorname{Spt}\left(\mu_{t}\right)$. Thus we can consider that $S_{t}$ is Lipschitz continuous and extend its definition domain to $\mathbb{R}^{d}$. 
2.1. Some particular cases. Let $t \in] 0,1\left[\right.$ and $F \subset \mathbb{R}^{d}$ satisfying one of the properties of Example 1.9. $\mathcal{H}^{d}(F)=0, \mathcal{H}^{d-1}(F)=0, \operatorname{dim} \mathcal{H}(F) \leq d-1$ or " $F$ is a $d-1$ dimensional Lipschitz piece". Consider $S_{t}(F)$ and observe that it satisfies the same property as $F$, just because $S_{t}$ is Lipschitz continuous. Thus if a transport-regular $\mu_{0}$ satisfies properties described in Example 1.9, $\mu_{t}$ satisfies the same properties and is transport-regular. On the contrary, in Example 1.10 the Lipschitz image of a $\mathcal{C}^{2}$ submanifold is not necessarily $\mathcal{C}^{2}$. This fact is at the origin of the proof of Theorem 2.2 .

2.2. A counterexample in the general case. The counterexample will take place in $\mathbb{R}^{2}$, and we will consider $t=1 / 2$. Generalizations to higher dimension and other interpolation parameters will be given in Theorem 2.5. We start with a result of one variable analysis.

Proposition 2.1. There exists a $\mathcal{C}^{1}$ function $f$ defined on $[0,1]$ such that for all $H \in \mathcal{C}^{2}([0,1])$,

$$
\mathcal{L}^{1}(\{x \in[0,1], f(x)=H(x)\})=0 .
$$

See [3, Appendix] for statements with Hölder conditions.

Proof. It is enough to prove that some continuous function $g$ meets the $C^{1}$ functions on a set of measure 0 . Actually if there exists a continuous $g$ satisfying

$$
\mathcal{L}^{1}(\{g=h\})=0
$$

for all $h \in C^{1}([0,1])$, we can set $f(x)=\int_{0}^{x} g$. Therefore for $H$ of class $\mathcal{C}^{2}$, at density points of $\{f=H\}$ we must have $f^{\prime}=H^{\prime}$ so that $\mathcal{L}^{1}(\{f=H\}) \leq \mathcal{L}^{1}\left(\left\{g=H^{\prime}\right\}\right)=0$. One can see that a Weierstrass-like function (take for instance a van der Waerden function) or almost surely a one dimensional Brownian path can serve as an example of $g$. Actually these functions are nowhere approximatively differentiable on $[0,1]$. See [8] and the references therein for these and other results.

Figure 1 represents the construction involved in the next (main) theorem.

Theorem 2.2. There exists $\mu_{0}, \mu_{1}$, transport-regular measures of $\mathcal{P}_{2}\left(\mathbb{R}^{2}\right)$, such that $\mu_{1 / 2}$ is not transport-regular.

Proof. Consider $f$ as in Proposition 2.1. Up to multiplication by a constant one can assume that $\left|f^{\prime}\right|<1$. We introduce $q^{+}: x \rightarrow(x, f(x))$ from $[0,1]$ to the graph of $f$, $q^{-}: x \rightarrow(x,-f(x))$ and $p:(x, y) \rightarrow x$. We now set $\mu_{0}=q_{\#}^{+} \nu$ and $\mu_{1}=q_{\#}^{-} \nu$, where $\nu$ denotes the restriction of the Lebesgue measure to $[0,1]$. Following subsection 1.2 .2 we denote by $\vec{u}$ the unit vector of the $x$-axis. As $\left|f^{\prime}\right|<1, \preceq$ is a total order on spt $\mu_{0}$ and spt $\mu_{1}$. It follows from Lemma 1.6 and the comment after it that $\pi=\left(q^{+} \otimes q^{-}\right)_{\#} \nu$ is the unique optimal transport plan between $\mu_{0}$ and $\mu_{1}$. But $\frac{q^{+}+q^{-}}{2}(x)=(x, 0)$ so that $\mu_{1 / 2}=\mathcal{L}_{\mid[0,1] \times\{0\}}^{1}$ is concentrated on the $x$-axis. Thus $\mu_{1 / 2}$ is not transport-regular (consider $\phi(x, y)=|y|$ in Proposition 1.7 or compare with Example 4.9 in [12]).

The last thing we have to check is that $\mu_{0}$ and $\mu_{1}$ are transport-regular. Of course it suffices to consider $\mu_{0}$. Note that for any measurable set $F \subset \mathbb{R}^{2}$ we have

$$
\mu_{0}(F)=\mathcal{L}^{1}\left(p\left(\operatorname{Spt}\left(\mu_{0}\right) \cap F\right)\right),
$$


where $\operatorname{Spt}\left(\mu_{0}\right)$ is simply the graph of $f$ in $\mathbb{R}^{2}$. Let $\phi$ be a convex function and $S$ the set of the non-differentiability points of $\phi$. We have to show $\mu_{0}(S)=0$. As Alberti proved (Proposition 1.8), there are 1 dimensional submanifolds of class $\mathcal{C}^{2}$, $M_{k} \subset \mathbb{R}^{d}$ and a set $N$ with $\mathcal{H}^{1}(N)=0$ such that $S \subset \bigcup_{k=1}^{+\infty} M_{k} \cup N$. But

$$
\mu_{0}(N)=\mathcal{L}^{1}\left(p\left(\operatorname{Spt}\left(\mu_{0}\right) \cap N\right)\right) \leq \mathcal{H}^{1}(p(N)) \leq \mathcal{H}^{1}(N)=0
$$

because $p$ is 1-Lipschitz. Let us now prove that $\mu_{0}\left(M_{k}\right)=0$ for a given $k \in \mathbb{N}$. Because of Sard's lemma, $\mathcal{H}^{1}\left(p\left(M_{k}^{u}\right)\right)=0$, where $M_{k}^{u}=\left\{m \in M_{k} \mid \vec{u}\right.$ is normal to $\left.M_{k}\right\}$. Therefore

$$
\mu_{0}\left(M_{k}^{u}\right)=\mathcal{L}^{1}\left(p\left(\operatorname{Spt}\left(\mu_{0}\right) \cap M_{k}^{u}\right)\right)=0 .
$$

Moreover, $M_{k} \backslash M_{k}^{u}$ is the countable union of pieces of graphs of class $\mathcal{C}^{2}$. Thus

$$
\mathcal{L}^{1}\left(p\left(\operatorname{Spt}\left(\mu_{0}\right) \cap\left(M_{k} \backslash M_{k}^{u}\right)\right)\right)=0
$$

just because $f$ follows the consequence of Proposition 2.1. Finally $\mu_{0}\left(M_{k}\right)=0$ and $\mu_{0}(S)=0$, as we want.

Remark 2.3. We have actually proven that $\mu_{0}$ satisfies the hypotheses of Example 1.10 .

Remark 2.4. The optimal transport map $T$ used in this construction is an orthogonal symmetry. This is a very special feature because for most measures usually considered in optimal transport theory, pushing forward by symmetry provides a transport plan that does not satisfy the cyclical monotonicity.

2.2.1. Generalization. In this last part we generalize Theorem 2.2 to higher dimensions $d$ and interpolation parameters $t \in] 0,1[$. The proof for $d>2$ can be deduced from $d=2$ thanks to a tensorization method.

Theorem 2.5. For any $d \geq 2$ and any $t \in] 0,1\left[\right.$, there exists $\mu_{0}, \mu_{1}$ transport-regular measures of $\mathcal{P}_{2}\left(\mathbb{R}^{d}\right)$ such that $\mu_{t}$ is not transport-regular.

First, we keep $t=1 / 2$. Let us define

$$
\mu_{i}^{(d)}=\underbrace{\nu \otimes \cdots \otimes \nu}_{d-2} \otimes \mu_{i}
$$

for $i=0,1$, where $\nu$ is the uniform measure on $[0,1]$ and $\mu_{i}$ is a measure of $\mathbb{R}^{2}$ as in Theorem 2.2. We claim that these measures are transport-regular. We show it for $\mu_{0}^{(d)}$. Observe that $\mu_{0}^{(d)}=q_{\#}^{+(d)} \nu^{\otimes d-1}$, where

$$
q^{+(d)}\left(x_{1}, \ldots, x_{d-1}\right)=\left(x_{1}, \ldots, x_{d-1}, f\left(x_{d-1}\right)\right),
$$

for the same $f: \mathbb{R} \rightarrow \mathbb{R}^{2}$ as used in Theorem [2.2 Actually $\mu_{0}^{(d)}$ satisfies the hypotheses of Example 1.10. On the one hand $\mu_{0}^{(d)}$ is absolutely continuous with respect to $\mathcal{H}^{d-1}$. To show this, just consider a relation corresponding to (2.3) where $p^{(d)}\left(x_{1}, \ldots, x_{d}\right)=\left(x_{1}, \ldots, x_{d-1}\right)$ replaces $p$. On the other hand, any hypersurface of class $\mathcal{C}^{2}$ can be decomposed into two parts. The first part $M^{\prime}$ is made of the points where the the normal vector is in $\mathbb{R}^{d-1} \times\{0\}$. Then because of Sard's lemma $\mathcal{L}^{d-1}\left(p^{(d)}\left(M^{\prime}\right)\right)=0$. The other part is a countable union of graph pieces of class $\mathcal{C}^{2}$. Let $M^{\prime \prime}$ be one of these pieces. Hence

$$
M^{\prime \prime}=\{(x, v(x)) \mid x \in U\},
$$


where $v \in \mathcal{C}^{2}(U, \mathbb{R})$ and $U \subset \mathbb{R}^{d-1}$ is an open set. As $v\left(x_{1}, \ldots, x_{d-2}, \cdot\right) \in \mathcal{C}^{2}(\mathbb{R})$ we can conclude using the proof of Theorem 2.2 and Fubini's theorem that

$$
\mathcal{L}^{d-1}\left(p^{(d)}\left(\operatorname{Spt}\left(\mu_{0}^{(d)}\right) \cap M^{\prime \prime}\right)\right)=0 .
$$

Thus $\mu_{0}$ is transport-regular.

We now prove that formula (2.4) also holds for $i \in] 0,1[$; i.e., the displacement interpolation commutes with the $\nu^{\otimes d-2}$-tensorization. From there we will be done because $\mu_{1 / 2}^{(d)}$ is concentrated on $\mathbb{R}^{d-1} \times\{0\}$ so that it is not transport-regular. Consider the optimal transport plan $\pi^{(d)}$ between $\mu_{0}^{(d)}$ and $\mu_{1}^{(d)}$. For $r$ defined by $r\left(x_{1}, \ldots, x_{d}\right)=\left(x_{d-1}, x_{d}\right)$ we obtain a coupling $\pi_{r}=(r \otimes r)_{\#} \pi^{(d)}$ between $\mu_{0}$ and $\mu_{1}$. This projection reduced the value of the cost in (1.1); we have $C\left(\pi_{r}\right) \leq C\left(\pi^{(d)}\right)$. But $C\left(\pi^{(d)}\right) \leq C\left(\left((\mathrm{Id} \otimes \mathrm{Id})_{\#} \nu\right)^{\otimes d-2} \otimes \pi\right)=C(\pi)$, where $\pi$ is the optimal transport plan between $\mu_{0}$ and $\mu_{1}$. Hence $\pi_{r}=\pi$. From there it is not difficult to see that

$$
\pi^{(d)}=\left((\mathrm{Id} \otimes \mathrm{Id})_{\#} \nu\right)^{\otimes d-2} \otimes \pi
$$

Note that in this proof we have been implicitly identifying $\mathbb{R}^{d} \times \mathbb{R}^{d}$ with $(\mathbb{R} \times \mathbb{R})^{d}$.

For the generalization in the convexity parameter $t$, observe that for any $T>1 / 2$, the non-transport-regular $\mu_{1 / 2}$ is the interpolated measure of parameter $t=\frac{1}{2 T}>$ $1 / 2$ between $\mu_{0}$ and $\mu_{T}$. Moreover, $\mu_{T}$ is transport-regular because $\mu_{T}=u_{\#}^{T} \mu_{1}$, where $u^{T}:\left(x_{1}, \ldots, x_{d}\right) \rightarrow\left(x_{1}, \ldots, x_{d-1}, 2\left(T-\frac{1}{2}\right) x_{d}\right)$ is affine and invertible. By a symmetric argument one can conclude this for $t<1 / 2$ too.

Remark 2.6. In the one dimensional case, transport-regular means with no atom. If $\mu_{0}$ and $\mu_{1}$ have no atom, it is the same for any transport plan $\pi$. For an optimal $\pi$, it follows from subsection 1.2.1 that $x<x^{\prime} \Rightarrow y \leq y^{\prime}$ for $(x, y),\left(x^{\prime}, y^{\prime}\right) \in \operatorname{Spt}(\pi)$. Hence for $t \in] 0,1\left[, e_{t}\right.$ is one-to-one when restricted to $\operatorname{Spt}(\pi)$, and $\mu_{t}$ has no atom.

\section{ACKNOWLEDGEMENTS}

The author wishes to thank Nicola Gigli and Benoît Kloeckner for reading the paper and for their editorial advice. He would also like to thank Shin-Ichi Ohta for Remark 0.1 and the referee for a careful reading.

\section{REFERENCES}

1. G. Alberti, On the structure of singular sets of convex functions, Calc. Var. Partial Differential Equations 2 (1994), no. 1, 17-27. MR1384392 (97e:26010)

2. L. Ambrosio, N. Gigli, and G. Savaré, Gradient flows in metric spaces and in the space of probability measures, second ed., Lectures in Mathematics ETH Zürich, Birkhäuser Verlag, Basel, 2008. MR2401600 (2009h:49002)

3. G. Anzellotti and R. Serapioni, $C^{k}$-rectifiable sets, J. Reine Angew. Math. 453 (1994), 1-20. MR 1285779 (95g:49078)

4. Y. Brenier, Polar factorization and monotone rearrangement of vector-valued functions, Comm. Pure Appl. Math. 44 (1991), no. 4, 375-417. MR.1100809 (92d:46088)

5. W. Gangbo and R. J. McCann, The geometry of optimal transportation, Acta Math. 177 (1996), no. 2, 113-161. MR.1440931 (98e:49102)

6. N. Gigli, On the inverse of Brenier-McCann theorems and the structure of $\left(\mathcal{P}_{2}, W_{2}\right)$, preprint (2009).

7. R. Jordan, D. Kinderlehrer, and F. Otto, The variational formulation of the Fokker-Planck equation, SIAM J. Math. Anal. 29 (1998), no. 1, 1-17. MR1617171(2000b:35258)

8. J. Malý and L. Zajíček, Approximate differentiation: Jarník points, Fund. Math. 140 (1991), no. 1, 87-97. MR:1139090 (92m:26006) 
9. R. J. McCann, A convexity principle for interacting gases, Adv. Math. 128 (1997), no. 1, 153-179. MR1451422 (98e:82003)

10. A. Petrunin, Parallel transportation for Alexandrov space with curvature bounded below, Geom. Funct. Anal. 8 (1998), no. 1, 123-148. MR1601854 (98j:53048)

11. C. Villani, Topics in optimal transportation, Graduate Studies in Mathematics, vol. 58, American Mathematical Society, Providence, RI, 2003. MR 1964483 (2004e:90003)

12. __ Optimal transport, Grundlehren der Mathematischen Wissenschaften, vol. 338, Springer-Verlag, Berlin, 2009. MR2459454 (2010f:49001)

13. L. Zajíček, On the differentiation of convex functions in finite and infinite dimensional spaces, Czechoslovak Math. J. 29(104) (1979), no. 3, 340-348. MR536060 (80h:46063)

Institut de Recherche Mathématique Avancée, UMr 7501, Université de Strasbourg et CNRS, 7 rue René Descartes, 67000 Strasbourg, France

E-mail address: nicolas.juillet@math.unistra.fr 